\title{
Duality for semiantichains and unichain coverings in products of special posets
}

\author{
Qi Liu and Douglas B. West*
}

\begin{abstract}
Saks and West conjectured that for every product of partial orders, the maximum size of a semiantichain equals the minimum number of unichains needed to cover the product. We prove the case where both factors have width 2 . We also use the characterization of product graphs that are perfect to prove other special cases, including the case where both factors have height 2 . Finally, we make some observations about the case where both factors have dimension 2 .
\end{abstract}

\section{Introduction}

In this paper, we study min-max relations involving natural subsets of partially ordered sets (posets). In a poset, chains and antichains are sets of pairwise comparable or pairwise incomparable elements, respectively. The height and width of a poset are the maximum sizes of chains and antichains, respectively.

Since every chain contains at most one element from an antichain, the number of chains needed to cover the elements of a poset is at least its width. Dilworth [4] proved that this many chains suffice in any finite poset. A chain partition of a poset using the fewest chains is a Dilworth decomposition.

Greene and Kleitman [7] generalized Dilworth's Theorem. A $k$-family in a poset is a family of elements containing no chain of size $k+1$. Equivalently, a $k$-family is a union of $k$ antichains. Every $k$-family has size at most $\sum_{i} \min \left\{k,\left|C_{i}\right|\right\}$, where $C_{1}, \ldots, C_{t}$ is a chain decomposition, because chain $C_{i}$ contributes at $\operatorname{most} \min \left\{k,\left|C_{i}\right|\right\}$ elements to a $k$-family. A chain decomposition for which this value equals the maximum size of a $k$-family is $k$ saturated. The Greene-Kleitman Theorem states that for every finite poset and every $k$, there is a $k$-saturated chain decomposition (in fact, one that is both $k$-saturated and $(k+1)$ saturated). Saks and West conjectured a generalization of the Greene-Kleitman Theorem; our goal here is to prove special cases of it.

*Department of Mathematics, University of Illinois, Urbana, IL 61801, west@math.uiuc.edu. Work supported in part by NSA Award No. H98230-06-1-0065. 
The product of posets $P$ and $Q$ is the poset $P \times Q$ whose elements are the ordered pairs $(p, q)$ with $p \in P$ and $q \in Q$, with $(p, q) \leq\left(p^{\prime}, q^{\prime}\right)$ in $P \times Q$ precisely when $p \leq p^{\prime}$ in $P$ and $q \leq q^{\prime}$ in $Q$. Given an expression of a poset as a product $P \times Q$, a semiantichain is a family in which elements are comparable only if they differ in both coordinates, and a unichain is a chain that is constant in one coordinate. Thus every antichain in $P \times Q$ is a semiantichain, and every unichain is a chain. Furthermore, every unichain contains at most one element from any semiantichain, so the number of unichains needed to cover a product is at least the maximum size of a semiantichain.

Saks and West conjectured the natural min-max relation: in every product of finite posets $P$ and $Q$, the maximum size of a semiantichain equals the minimum size of a unichain covering. We call this the Semiantichain Conjecture; it reduces to the Greene-Kleitman Theorem when $P$ or $Q$ is a chain. Other special cases for posets having $k$-saturated chain partitions with special properties were presented in $[12,13,14,15]$.

In this paper, we prove the conjecture for the special case where both posets have width 2, using structural counting arguments.

In graph-theoretic language, the statement of the conjecture is weaker than the statement that the cartesian product of the comparability graphs of the two posets is a perfect graph. We use the characterization $[3,11]$ of cartesian product graphs that are perfect to prove the conjecture for two more cases. These are the case where both posets have height 2 and the case where one poset is a ranking and the comparability graph of the other is a tree.

Finally, we offer an observation on the case where both factors have dimension 2.

\section{Products of width-2 posets}

To motivate our proof for products of width-2 posets, we present a famous example.

Example 2.1 The "Greene-Kleitman poset" $R$ is shown in Figure 1. It has width 2, as demonstrated by the decomposition into two 3-element chains, and it has another natural partition into chains of sizes 4,1,1. The first partition is 1 -saturated and 2 -saturated but not 3-saturated; the second is 2-saturated and 3-saturated but not 1-saturated.

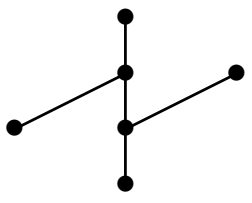

Figure 1: The Greene-Kleitman poset $R$

A maximum $k$-family in a poset $P$ is a maximum union of $k$ antichains. Since the elements of a $k$-element chain (written $\underline{k}$ ) are pairwise comparable, this also corresponds to 
a maximum semiantichain in $P \times \underline{k}$. On the other hand, $k$-saturated partitions lead to upper bounds, and they generate unichain coverings of $P \times \underline{k}$.

The poset $R$ has width 2 . In comparing $R \times \underline{1}$ and $R \times \underline{3}$, the chain partitions of $R$ used to generate optimal unichain decompositions differ: one is the Dilworth decomposition, and the other is a longest chain together with a Dilworth decomposition of the rest of the poset. This phenomenon will arise in general for products of width-2 posets; which of the two partitions should be used to generate a unichain covering will depend on comparison of various parameters of the two posets.

Next we discuss simple ways to form semiantichains and unichain coverings from antichain and chain partitions of the two factor posets.

When $A$ and $B$ are antichains in $P$ and $Q$, the set of elements formed by pairing each element of $A$ with each element of $B$ is a semiantichain in $P \times Q$; each element from one poset is paired with an antichain from the other. Indeed, when $A_{1}, \ldots, A_{h}$ and $B_{1}, \ldots, B_{h}$ are antichain decompositions of $P$ and $Q$ (with trailing empty antichains added as needed), any pairing of $A_{i}$ with $B_{\sigma(i)}$ for all $i$ produces a semiantichain of size $\sum_{i=1}^{m}\left|A_{i}\right|\left|B_{\sigma(i)}\right|$. A semiantichain produced in this way is a decomposable semiantichain.

Algebraic manipulation shows that the size of this semiantichain is maximized by pairing the antichains in decreasing order of size in each decomposition. That is, letting $\mathcal{A}=$ $A_{1}, \ldots, A_{h}$ and $\mathcal{B}=B_{1}, \ldots, B_{h}$, with $\left|A_{1}\right| \geq \cdots \geq\left|A_{h}\right|$ and $\left|B_{1}\right| \geq \cdots \geq\left|B_{h}\right|$, the largest decomposable semiantichain produced from these decompositions has size $\sum_{i=1}^{m}\left|A_{i}\right|\left|B_{i}\right|$; call this value $g(\mathcal{A}, \mathcal{B})$, where " $g$ " connotes "greedy".

Similarly, a natural notion of decomposable unichain covering arises from chain decompositions $C_{1}, \ldots, C_{r}$ and $D_{1}, \ldots, D_{s}$ of $P$ and $Q$. For each pair $\left(C_{i}, D_{j}\right)$, we cover $C_{i} \times D_{j}$ by using $\min \left\{\left|C_{i}\right|,\left|D_{j}\right|\right\}$ copies of the longer chain. The size of the resulting unichain covering is $\sum_{i, j} \min \left\{\left|C_{i}\right|,\left|D_{j}\right|\right\}$; call this value $m(\mathcal{C}, \mathcal{D})$, where $\mathcal{C}=C_{1}, \ldots, C_{r}$ and $\mathcal{D}=D_{1}, \ldots, D_{s}$.

Always $m(\mathcal{C}, \mathcal{D}) \geq g(\mathcal{A}, \mathcal{B}) ;[14]$ obtained conditions under which equality holds. We will use decomposable seminantichains and unichain coverings in proving the theorem.

We begin by producing the needed antichain partitions. It is a standard observation that iteratively removing the antichain of maximal remaining elements in a poset $P$ yields the min-max relation that the minimum size of an antichain decomposition of $P$ equals the maximum size of a chain in $P$ (this is a "dual" of Dilworth's Theorem).

Lemma 2.2 An n-element poset with width 2 and height $h$ has $n-h$ pairwise disjoint 2element antichains. If $P$ has width 2, size $n$, and height $n-r$, and $P^{\prime}$ has width 2 , size $n^{\prime}$, and height $n^{\prime}-r^{\prime}$, then $P \times P^{\prime}$ has a semiantichain of size $g\left(\mathcal{A}, \mathcal{A}^{\prime}\right)$, where $\mathcal{A}$ has $r$ antichains of size 2 and $n-2 r$ antichains of size 1 , and $\mathcal{A}^{\prime}$ has $r^{\prime}$ antichains of size 2 and 
$n^{\prime}-2 r^{\prime}$ antichains of size 1 . Furthermore, with $r \leq r^{\prime}$ (by symmetry),

$$
g\left(\mathcal{A}, \mathcal{A}^{\prime}\right)= \begin{cases}n^{\prime}+2 r & \text { if } n-r \geq n^{\prime}-r^{\prime}, \\ n+r+r^{\prime} & \text { if } r^{\prime} \leq n-r \leq n^{\prime}-r^{\prime}, \\ 2 n & \text { if } n-r \leq r^{\prime} .\end{cases}
$$

Proof. Since the largest chain has $h$ elements, there is a partition of $P$ into $h$ antichains. Since each antichain has size at most 2, every such partition consists of $n-h$ antichains of size 2 and $2 h-n$ antichains of size 1 .

Thus the specified antichain partitions $\mathcal{A}$ and $\mathcal{A}^{\prime}$ exist, and they produce the specified decomposable semiantichain. To compute its size, we consider three cases.

$$
\begin{aligned}
& \text { List of Sizes } \\
& r \quad n-2 r \quad \text { Case Computation of } g\left(\mathcal{A}, \mathcal{A}^{\prime}\right) \\
& \mathcal{A}: 2 \cdots 21 \cdots 1 \quad n^{\prime}-r^{\prime} \leq n-r \quad 4 r+2\left(r^{\prime}-r\right)+n^{\prime}-2 r^{\prime}=2 r+n^{\prime} \\
& \mathcal{A}^{\prime}: 2 \cdots 21 \cdots 1 \quad r^{\prime} \leq n-r \leq n^{\prime}-r^{\prime} \quad 4 r+\left(n-2 r-r^{\prime}\right)+\left(r^{\prime}-r\right)=n+r+r^{\prime} \\
& r^{\prime} \quad n^{\prime}-2 r^{\prime} \quad n-r \leq r^{\prime} \quad 4 r+2(n-2 r)=2 n
\end{aligned}
$$

We will show that the decomposable semiantichain produced from $\mathcal{A}$ and $\mathcal{A}^{\prime}$ in Lemma 2.2 is a maximum semiantichain, by providing unichain decompositions of the same size. The three cases use different unichain decompositions.

In computing the sizes of the unichain coverings, we use two remarks. Since $m(\mathcal{C}, \mathcal{D})$ depends only on the sizes of the chains, we instead write $m\left(c_{1}, \ldots, c_{r} ; d_{1}, \ldots, d_{s}\right)$, where the chains in $\mathcal{C}$ have sizes $c_{1}, \ldots, c_{r}$ and the chains in $\mathcal{D}$ have sizes $d_{1}, \ldots, d_{s}$. Also, the computation is monotone in the sizes; for example, replacing $c_{1}$ with an arbitrarily large number, which we may represent as $\infty$, cannot reduce the value.

Theorem 2.3 If $P$ and $P^{\prime}$ are posets of width 2, then the maximum size of a semiantichain in $P \times P^{\prime}$ equals the minimum size of a unichain covering of $P \times P^{\prime}$.

Proof. We use $n, n-r, n^{\prime}, n^{\prime}-r^{\prime}$ for the size and height of $P$ and $P^{\prime}$ as in Lemma 2.2, with $r \leq r^{\prime}$. In each case, we use the decomposable semiantichain in $P \times P^{\prime}$ generated from $\mathcal{A}$ and $\mathcal{A}^{\prime}$ as in Lemma 2.2. We seek a unichain covering of (at most) the same size.

Let $a$ and $b$ with $a+b=n$ be the sizes of the chains in some Dilworth decomposition of $P$. Let $L$ be a chain of size $n-r$ in $P$. The poset $P-L$ of size $r$ also has width at most 2 . Let $k$ and $l$ with $k+l=r$ be the sizes of the chains in a Dilworth decomposition of $P-L$ (one of these chains may be empty). We have defined two chain decompositions of $P$; they are $\mathcal{C}$ with sizes $(a, b)$ and $\mathcal{D}$ with sizes $(n-r, k, l)$. Similarly, $P^{\prime}$ has two chain partitions: $\mathcal{C}^{\prime}$ with sizes $\left(a^{\prime}, b^{\prime}\right)$ and $\mathcal{D}^{\prime}$ with sizes $\left(n^{\prime}-r^{\prime}, k^{\prime}, l^{\prime}\right)$.

Case 1: $n-r \geq n^{\prime}-r^{\prime}$. We use the covering generated by $\mathcal{D}$ and $\mathcal{C}^{\prime}$ :

$$
\begin{aligned}
m\left(n-r, k, l ; a^{\prime}, b^{\prime}\right) & =m\left(n-r ; a^{\prime}, b^{\prime}\right)+m\left(k, l ; a^{\prime}, b^{\prime}\right) \\
& \leq m\left(\infty ; a^{\prime}, b^{\prime}\right)+m(k, l ; \infty, \infty)=n^{\prime}+2 r=g\left(\mathcal{A}, \mathcal{A}^{\prime}\right) .
\end{aligned}
$$


Case 2: $r^{\prime} \leq n-r \leq n^{\prime}-r^{\prime}$. Partition $P \times P^{\prime}$ into two subposets $L \times P^{\prime}$ and $(P-L) \times P^{\prime}$. Cover $L \times P^{\prime}$ using the covering generated by $L$ and $\mathcal{D}^{\prime}$. Cover $\left(P_{1}-L\right) \times P_{2}$ using the covering generated by $\mathcal{D}-\{L\}$ and $\mathcal{C}^{\prime}$. We compute the total number of unichains, using the given relations $k^{\prime}+l^{\prime}=r^{\prime} \leq n-r$.

$$
\begin{aligned}
m\left(n-r ; n^{\prime}-r^{\prime}, k^{\prime}, l^{\prime}\right)+m\left(k, l ; a^{\prime}, b^{\prime}\right) & \leq m\left(n-r ; \infty, k^{\prime}, l^{\prime}\right)+m(k, l ; \infty, \infty) \\
& =n-r+k^{\prime}+l^{\prime}+2(k+l)=n+r+r^{\prime}=g\left(\mathcal{A}, \mathcal{A}^{\prime}\right) .
\end{aligned}
$$

Case 3: $n-r \leq r^{\prime}$. We use the covering generated by $\mathcal{C}$ and $\mathcal{C}^{\prime}$

$$
m\left(a, b ; a^{\prime}, b^{\prime}\right) \leq m(a, b ; \infty, \infty)=2(a+b)=2 n=g\left(\mathcal{A}, \mathcal{A}^{\prime}\right)
$$

In each case, we have found a unichain covering with size at most $g\left(\mathcal{A}, \mathcal{A}^{\prime}\right)$.

\section{Semiantichains and perfect graphs}

The Semiantichain Conjecture can be phrased as a statement about products of comparability graphs. The comparability graph of a poset has a vertex for each element, with vertices adjacent when the elements are comparable. For a graph $G$, let $\alpha(G), \omega(G), \chi(G), \theta(G)$ denote, respectively, the maximum size of an independent set of vertices, the maximum size of a clique (a set of pairwise adjacent vertices), the chromatic number, and the minimum number of cliques needed to cover the vertices.

A graph $G$ is perfect if $\chi(H)=\omega(H)$ for each induced subgraph $H$. Lovász [9] proved that $G$ is perfect if and only if its complement $\bar{G}$ is perfect. The statement of perfection for $\bar{G}$ is that $\alpha(H)=\theta(H)$ for each induced subgraph of $G$. In the comparability graph of a poset $P$, each chain becomes a clique and each antichain becomes an independent set. Thus Dilworth's Theorem is equivalent to the statement that comparability graphs are perfect.

The (cartesian) product of graphs $G$ and $H$ is the graph $G \square H$ with vertex set $V(G) \times V(H)$ defined by putting $(u, v)$ adjacent to $\left(u^{\prime}, v^{\prime}\right)$ if $(1) u=u^{\prime}$ and $v v^{\prime} \in E(H)$ or $(2) v=$ $v^{\prime}$ and $u u^{\prime} \in E(G)$. Thus the product of the comparability graphs of $P$ and $Q$ is the "unicomparability graph" of $P \times Q$, with vertices adjacent when the corresponding elements lie together in a unichain.

The Semiantichain Conjecture is thus the statement that $\alpha(G \square H)=\theta(G \square H)$ when $G$ and $H$ are comparability graphs. Chappell [1] studied the conjecture from this viewpoint. The desired statement is weaker than saying that $G \square H$ is perfect, since the equality is needed only for the full graph. Indeed, rarely is the product of comparability graphs perfect.

Nevertheless, the Conjecture holds automatically when the product of the comparability graphs actually is perfect, so it is sensible to characterize when this holds. We use an old characterization of when the product of two perfect graphs is perfect. 
A Berge graph is a graph having no odd cycle or its complement as an induced subgraph. Since odd cycles and their complements are not perfect, every perfect graph is a Berge graph. Berge's famous Strong Perfect Graph Conjecture that every Berge graph is perfect was proved by Chudnovsky, Robertson, Seymour, and Thomas [2] after more than 40 years.

A graph is $H$-free if it has no induced subgraph isomorphic to $H$. A graph with four vertices and five edges is a diamond (also called kite). A graph with no induced cycle of length at least 4 is chordal (formerly called triangulated. A graph is a TDF graph if it is triangulated and diamond-free.

A 4-vertex graph having a triangle plus one "pendant" edge is a paw [10]; de Werra and Hertz [3] called it a "flag". They defined a graph to be a BFF graph if it is a Berge graph that is flag-free. In light of the Strong Perfect Graph Theorem, we may equivalently define a BFF graph to be a paw-free perfect graph. Furthermore, Olariu [10] proved that a graph is paw-free if and only if each component is triangle-free or is a complete multipartite graph.

These families have been used to characterize the pairs of graphs whose products are perfect. The characterization was found by Ravindra and Parthasarathy [11], and a complete proof appears in [3].

Theorem 3.1 If $G$ and $H$ are connected graphs with at least three vertices, then $G \square H$ is perfect if and only if one of the following mutually exclusive conditions holds:

(a) both are bipartite;

(b) one is a TDF graph and the other is a complete graph;

(c) one is a BFF graph with an induced diamond and the other is a tree.

A ranking or weak order is a poset whose elements can be partitioned into sets $P_{1}, \ldots, P_{m}$ such that $x<y$ for $x \in P_{i}$ and $y \in P_{j}$ if and only if $i<j$.

Corollary 3.2 The Semiantichain Conjecture holds for products of posets with height 2 and for products of posets in which one is a ranking and the other is a poset of height 2 whose comparability graph has no cycles.

Proof. We consider the consequences of Theorem 3.1.

Case a: A comparability graph is bipartite if and only if the poset has height 2 , so the conjecture holds for products of posets with height 2 .

Case b: A comparability graph is a complete graph if and only if the poset is a chain. We do not bother to characterize the posets whose comparability graphs are TDF graphs, since the conjecture always holds when one poset is a chain, by the Greene-Kleitman Theorem.

Case c: The comparability graph is a tree only for special posets with height 2, those having no cycle in the diagram. When the comparability graph is a BFF graph with an induced diamond, it has a triangle, and hence by the result of [10] it is a complete multipartite graph with at least three parts, which occurs precisely when the poset is a "ranking" with more than two levels. 


\section{Products of dimension-2 posets}

An extension of a poset $P$ is a poset $P^{\prime}$ with the same elements of $P$ such that if $x<y$ in $P$, then $x<y$ in $P^{\prime}$. A linear extension is an extension that is a chain. A realizer of $P$ is a set $\mathcal{L}$ of extensions of $P$ such that $x<y$ in $P$ if and only if $x<y$ in every member of $\mathcal{L}$. The dimension $\operatorname{dim} P$ is the minimum size of a realizer of $P$ by linear extensions. Dushnik and Miller [5] proved that a poset has dimension 2 if and only if the complement of its comparability graph is also a comparability graph.

Proving the conjecture of Saks and West for posets of dimension 2 would strengthen Theorem 2.3, since the dimension of a poset is bounded by its width $[4,8]$. We studied a much smaller question that still remains open. Suppose that $P, P^{\prime}, Q$, and $Q^{\prime}$ are posets with dimension 2 such that the comparability graphs of $P^{\prime}$ and $Q^{\prime}$ are the complements of the comparability graphs of $P$ and $Q$, respectively. If the conjecture holds for $P \times Q$, then must it also hold for $P^{\prime} \times Q^{\prime}$ ?

The Ferrers diagram of a partition of $n$ consists of a left-justified array of $n$ dots, with $\lambda_{i}$ dots in row $\mathrm{i}$, where $\lambda_{i}$ is the $i$ th largest part of the partition. We write $\lambda=\left\{\lambda_{1}, \ldots, \lambda_{k}\right\}$, where $\lambda_{1} \geq \cdots \geq \lambda_{k}$. The conjugate $\lambda^{*}$ of an integer partition $\lambda$ is the partition whose Ferrers diagram is the transpose of the Ferrers diagram of $\lambda$. Write $\lambda^{*}=\left\{\lambda_{1}^{*}, \ldots, \lambda_{m}^{*}\right\}$, where $\lambda_{1}^{*} \geq \cdots \geq \lambda_{m}^{*}$. Note that $k=\lambda_{1}^{*}$ and $m=\lambda_{1}$. Consider two partitions $\lambda$ and $\mu$, and let $\lambda^{*}$ and $\mu^{*}$ be their conjugates, respectively. Observe that $\sum_{i, j} \min \left\{\left|\lambda_{i}\right|,\left|\mu_{j}\right|\right\}=\sum_{i} \lambda_{i}^{*} \mu_{i}^{*}$.

Given a poset $P$, let $d_{k}(P)$ denote the maximum size of a $k$-family in $P$, and let $\Delta_{k}^{P}=$ $d_{k}(P)-d_{k-1}(P)$. (The notation $d_{k}$ honors Dilworth.) Let $\Delta^{P}=\left\{\Delta_{1}^{P}, \Delta_{2}^{P}, \ldots\right\}$. A $k$-cofamily in $P$ is a set of elements containing no antichain of size $k+1$; by Dilworth's Theorem, a $k$ cofamily is a union of $k$ chains. Let $\hat{d}_{k}(P)$ denote the maximum size of a $k$-cofamily in $P$, and let $\hat{\Delta}_{k}^{P}=\hat{d}_{k}(P)-\hat{d}_{k-1}(P)$. Let $\hat{\Delta}^{P}=\left\{\hat{\Delta}_{1}^{P}, \hat{\Delta}_{2}^{P}, \ldots\right\}$. For every poset $P$, Greene [6] proved that $\Delta^{P}$ and $\hat{\Delta}^{P}$ are conjugate partitions of the integer $|P|$. Thus when $P$ and $P^{\prime}$ are posets whose comparability graphs are complementary, we say that $P^{\prime}$ is a conjugate poset of $P$.

Let $\Delta^{P} \cdot \Delta^{Q}=\sum_{k} \Delta_{k}^{P} \Delta_{k}^{Q}$. West and Tovey [14] proved that always $m(\mathcal{C}, \mathcal{D}) \geq \Delta^{P} \cdot \Delta^{Q} \geq$ $g(\mathcal{A}, \mathcal{B})$, where $\mathcal{A}$ and $\mathcal{B}$ are antichain partitions and $\mathcal{C}$ and $\mathcal{D}$ are chain partitions of $P$ and $Q$, respectively. A greedy antichain partition of a poset $P$ is an antichain partition $\mathcal{A}$ such that the union of $k$ largest antichains in $\mathcal{A}$ is a maximum $k$-family in $P$.

Proposition 4.1 If $P^{\prime}$ and $Q^{\prime}$ are conjugates of posets $P$ and $Q$ of dimension 2 having greedy antichain partitions, then $P^{\prime} \times Q^{\prime}$ has a decomposable unichain covering of size $\Delta^{P^{\prime}} \cdot \Delta^{Q^{\prime}}$.

Proof. The hypothesis yields antichain partitions for $P$ and $Q$ with size lists $\Delta^{P}$ and $\Delta^{Q}$, respectively. These antichain partitions become chain partitions in $P^{\prime}$ and $Q^{\prime}$. Thus $P^{\prime} \times Q^{\prime}$ has a decomposable unchain covering of size $m\left(\Delta^{P}, \Delta^{Q}\right)$. We compute

$$
m\left(\Delta^{P}, \Delta^{Q}\right)=\sum_{i, j} \min \left\{\Delta_{i}^{P}, \Delta_{j}^{Q}\right\}=\sum_{k} \hat{\Delta}_{k}^{P} \hat{\Delta}_{k}^{Q}=\sum_{k} \Delta_{k}^{P^{\prime}} \Delta_{k}^{Q^{\prime}}=\Delta^{P^{\prime}} \cdot \Delta^{Q^{\prime}}
$$


Similarly, let a greedy chain partition be a chain partition $\mathcal{C}$ such that for all $k$ the union of $k$ largest chains in $\mathcal{C}$ is a maximum $k$-cofamily. The size list of a greedy chain partition of $P$ is $\hat{\Delta}^{P}$. An analogue of the preceding proposition holds.

Proposition 4.2 If $P^{\prime}$ and $Q^{\prime}$ are conjugates of posets $P$ and $Q$ of dimension 2 having greedy chain partitions, then the product $P^{\prime} \times Q^{\prime}$ has a decomposable semiantichain of size $\Delta^{P^{\prime}} \cdot \Delta^{Q^{\prime}}$.

Proof. The hypothesis yields chain partitions for $P$ and $Q$ with size lists $\hat{\Delta}^{P}$ and $\hat{\Delta}^{Q}$, respectively. These chain partitions become antichain partitions in $P^{\prime}$ and $Q^{\prime}$. Since $\hat{\Delta}^{P}=$ $\Delta^{P^{\prime}}$ and $\hat{\Delta}^{Q}=\Delta^{Q^{\prime}}$, the product $P^{\prime} \times Q^{\prime}$ thus has a decomposable semiantichain of size $g\left(\Delta^{P^{\prime}}, \Delta^{Q^{\prime}}\right)$, which equals $\Delta^{P^{\prime}} \cdot \Delta^{Q^{\prime}}$.

One would hope that if $P$ and $Q$ are posets of dimension 2 having greedy chain partitions, then the product of their conjugates $P^{\prime}$ and $Q^{\prime}$ has a decomposable semiantichain of size $\Delta^{P^{\prime}} \cdot \Delta^{Q^{\prime}}$. Unfortunately, this is not true.

Example 4.3 Consider the Greene-Kleitman poset $R$ from Example 2.1. It has an antichain partition with size list $\{2,2,1,1\}$ and two chain partitions with size lists $\{4,1,1\}$ and $\{3,3\}$. In $R \times R$, we have $\Delta^{R} \cdot \Delta^{R}=2 \cdot 2+2 \cdot 2+1 \cdot 1+1 \cdot 1=10$. Since $m(\{4,1,1\},\{3,3\})=10$ and $g(\{2,2,1,1\},\{2,2,1,1\})=10$, there are decomposable unichain coverings and semiantichains of size $\Delta^{R} \cdot \Delta^{R}$. Note that $m(\mathcal{C}, \mathcal{D})=\Delta^{R} \cdot \Delta^{R}$ is achievable even though $R$ does not have a greedy chain partition.

In $R^{\prime} \times R^{\prime}$, we have $\Delta^{R^{\prime}} \cdot \Delta^{R^{\prime}}=4 \cdot 4+2 \cdot 2=20$. Since $m(\{2,2,1,1\},\{2,2,1,1\})=20$, the product has an decomposable unichain covering of size $\Delta^{R^{\prime}} \cdot \Delta^{R^{\prime}}$. However, $R^{\prime}$ has no greedy antichain partition. The size lists for antichain partitions are $\{4,1,1\}$ and $\{3,3\}$, but we have $g(\{4,1,1\},\{4,1,1\})=18, g(\{3,3\},\{3,3\})=18$, and $g(\{4,1,1\},\{3,3\})=15$.

Although the decomposable semiantichains of size 18 have size less than $\Delta^{R^{\prime}} \cdot \Delta^{R^{\prime}}$, in fact they are optimal, because $R^{\prime} \times R^{\prime}$ decomposes into 18 unichains, each of size 2 .

\section{References}

[1] G. G. Chappell, Optimization in Product of Combinatorial Structures, Ph.D. Thesis, University of Illinois (1996).

[2] M. Chudnovsky, N. Robertson, P. Seymour, and R. Thomas, The strong perfect graph theorem. Ann. of Math. (2) 164 (2006), 51-229.

[3] D. de Werra and A. Hertz, On perfectness of sums of graphs, Discrete Mathematics 195 (1999) 93-101. 
[4] R. P. Dilworth, A decomposition theorem for partially ordered sets. Ann. of Math. (2) 51 (1950), 161-166.

[5] B. Dushnik, and E. W. Miller, Partially ordered sets. Amer. J. Math. 63 (1941), 600-610.

[6] C. Greene, Some partitions associated with a partially ordered set. J. Combin. Theory A 20 (1976), 69-79.

[7] C. Greene and D. J. Kleitman, The structure of Sperner $k$-families, J. Combin. Theory A 20 (1976), 41-68.

[8] T. Hiraguchi, On the dimension of orders. Sci. Rep. Kanazawa Univ. 4 (1955), 1-20.

[9] L. Lovász, Normal hypergraphs and the perfect graph conjecture. Discrete Math. 2 (1972), 253-267.

[10] S. Olariu, Paw-free graphs. Inform. Process. Lett. 28 (1988), 53-54.

[11] G. Ravindra and K. R. Parthasarathy, Perfect product graphs. Discrete Math. 20 (1977/78), 177-186.

[12] C. A. Tovey and D. B. West, Networks and chain coverings in partial orders and their products, Order 2 (1985), 49-60.

[13] D. B. West, Unichain coverings in partial orders with the nested saturation property, (Special issue: ordered sets, Oberwolfach, 1985) Discrete Math. 63 (1987), 297-303.

[14] D. B. West and C. A. Tovey, Semiantichains and unichain coverings in direct products of partial orders, SIAM J. Algebraic Discrete Methods 2 (1981), 295-305.

[15] C. Wu, On relationships between semi-antichains and unichain coverings in discrete mathematics. Chinese Quart. J. Math. 13 (1998), 44-48. 